\title{
Internal wave transmission through a thermohaline staircase
}

\author{
Bruce R. Sutherland* \\ Department of Physics and Department of Earth \& Atmospheric Sciences, University of Alberta, \\ Edmonton, Alberta, Canada T6G 2E1
}

(Received 14 January 2016; published 9 May 2016)

\begin{abstract}
With the aim to predict energy transport by internal waves incident upon observed density staircases in the ocean, the theory for internal wave tunneling through a single finite-size mixed region is extended to predict the transmission of internal waves through a piecewise-constant density profile with an arbitrary number of steps. An analytic solution is found if all steps have equal vertical extent. From this solution, bounds are established for the relative incident horizontal wave number and frequency of waves that have negligible transmission, a result that is independent of the number of steps. If the step sizes vary randomly about a mean vertical extent, the transmission can be computed numerically for several random realizations. Provided the horizontal wavelength is much longer than the total vertical extent of the staircase, the standard deviation of the computed transmission coefficients is small and the mean agrees well with the analytic prediction for equally spaced steps. The results are applied to thermohaline staircases observed in the ocean in order to put lower bounds on the wavelengths of long inertia gravity waves that significantly transmit to great depth.
\end{abstract}

DOI: 10.1103/PhysRevFluids.1.013701

\section{INTRODUCTION}

Through double diffusion, the density stratification of the ocean, particularly at the thermocline, can evolve to have a staircase profile characterized by regions of near-uniform density separated by sharp density jumps [1]. This has been observed in warm regions such as the Tyrrhenian Sea in the Mediterranean [2,3] and in the western Atlantic near the Caribbean Sea [4-6]. Recent observations have also shown that density staircases persist over large regions of the Arctic Ocean [7-10]. It is this case that is of particular interest because the reduction in sea ice in recent years has resulted in enhanced generation of internal gravity waves (or internal waves, for short) [11,12]. Because internal waves move under the influence of buoyancy [13], it is unclear to what extent they can penetrate through well-mixed uniform-density regions.

An early examination of internal waves interacting with a thermohaline staircase was performed by Salusti [14], who examined the splitting of modes as was done for an effective two-layer system by Ref. [15]. This eigenvalue approach is analogous to the derivation for the band structure of electrons in a one-dimensional lattice. A mathematical approach more relevant to the problem of downward-propagating internal waves incident upon a thermohaline staircase is that followed first by Sutherland and Yewchuk [16], who predicted the transmission and reflection of internal waves analogous to the problem of electron tunneling across a potential barrier. They showed that internal waves can partially transmit across a uniform-density region (where the waves are evanescent) provided it is sufficiently thin compared to the horizontal wavelength. Furthermore, if the uniform-density region is flanked by density jumps (as would result from localized mixing of the background stratification), then enhanced transmission can occur for incident waves whose horizontal wave number and frequency are nearly resonant with those of the interfacial waves on either flank of the mixed patch. This analytic study was extended to examine the influence of shear [17]. Further numerical studies examined the transmission of waves through more complex

*bruce.sutherland@ualberta.ca; www.ualberta.ca/ bsuther 
stratification [18-22] and large-amplitude effects were also considered [23]. However, in all these studies except Ref. [22] the background stratification considered involved far fewer layers than occurs in a typical thermohaline staircase. Ghaemsaidi et al. [22] in part considered the transmission of waves across two well-mixed regions bounded by finite-depth stratified layers through theory, numerics, and experiments. They found that incident waves passing through multiple layers could result in transmission spikes in incident vertical wave number and frequency space occurring as a result of interference patterns between evanescent waves within the mixed regions and propagating waves within the stratified layers. Numerically they extended this work to examine the passage of waves through multiple steps in a density staircase observed in the Arctic Ocean, with each step on the order of $1 \mathrm{~m}$ deep. They found significantly reduced transmission of energy by incident waves with frequency marginally larger than the Coriolis frequency, particularly for waves with vertical wavelengths on the order of tens of meters. Transmission increased monotonically toward unity for incident waves with vertical wavelength larger than $100 \mathrm{~m}$. The study pointed to the nontrivial effect of staircase stratification upon wave transmission as it depends upon the frequency and wave number of the incident waves and has inspired the extension of their theory presented here that predicts internal wave transmission through an arbitrary number of steps.

Specifically, this theoretical-numerical study predicts the transmission of internal waves across an arbitrary number of mixed regions separated by discontinuous density jumps. An analytic formula is found in the case for which each step has the same depth $L$. The sensitivity of this result to nonuniform step sizes is also examined numerically. From the analytic solutions, bounds are found that set the wavelength and frequency of incident internal waves that can pass through the staircase.

After describing the mathematical formulation of the problem in Sec. II, analytic solutions are found in Sec. III for the case of a staircase having an arbitrary number of evenly spaced steps. The case of an uneven staircase is examined numerically in Sec. IV, in which transmission is found for several stochastic representations of the density staircase. The case of varying density jumps is also briefly considered. In Sec. V the results are summarized and applied to a typical oceanographic circumstance.

\section{PROBLEM SETUP}

The transmission of internal waves across a density staircase is computed for small-amplitude two-dimensional waves in inviscid Boussinesq fluid. The background is taken to be stationary with density profile $\bar{\rho}(z)$ characterized by $J$ uniform-density steps, each of mean depth $L$. Conceptually, it is imaged that a staircase of total vertical extent $J L$ has developed, for example, due to double diffusion, within a uniformly stratified region characterized by buoyancy frequency $N_{0}$. Explicitly,

$$
\bar{\rho}(z)= \begin{cases}\rho_{00}\left(1-\frac{\Delta \rho}{\rho_{00}} \frac{z}{L}\right), & z>0 \\ \rho_{00}\left[1+\left(j-\frac{1}{2}\right) \frac{\Delta \rho}{\rho_{00}}\right], & -L\left(j+\epsilon \sigma_{j}\right)<z<-L\left(j-1+\epsilon \sigma_{j-1}\right), j=1,2, \ldots, J \\ \rho_{00}\left(1-\frac{\Delta \rho}{\rho_{00}} \frac{z}{L}\right), & z<-J L\end{cases}
$$

in which $\rho_{00}=\bar{\rho}\left(0^{+}\right)$is the characteristic density. For simplicity, whether the vertical extent of each step is fixed or variable, the density jump between steps is fixed to be $\Delta \rho$ within the staircase and the density jump between the bounding uniformly stratified regions (for $z>0$ and $z<-J L$ ) and the first and last step is $\Delta \rho / 2$. Outside the staircase the density gradient has magnitude $\Delta \rho / L$. Hence the squared buoyancy frequency is $N_{0}^{2}=g^{\prime} / L$ in which $g^{\prime}=g \Delta \rho / \rho_{00}$ is the reduced gravity. The value of $\epsilon(0 \leqslant \epsilon<0.5)$ is a measure of the fluctuation of the step extent about the mean value $L$. For $1 \leqslant j<J, \sigma_{j}$ are random numbers between -1 and 1 but $\sigma_{0}$ and $\sigma_{J}$ are both zero.

The case with $\epsilon=0$ corresponds to every step having equal depth $L$, as shown in the left-hand plot of Fig. 1. Explicit analytic predictions of the transmission coefficient can be found for this symmetric circumstance. In cases with positive $\epsilon$, the depths of each step fluctuate about a mean value $L$, as illustrated in the right-hand plot of Fig. 1 . In this case the transmission coefficient can be computed numerically for a given set of random variables $\left\{\sigma_{j} \mid j=1, \ldots, J-1\right\}$. Repeating this 

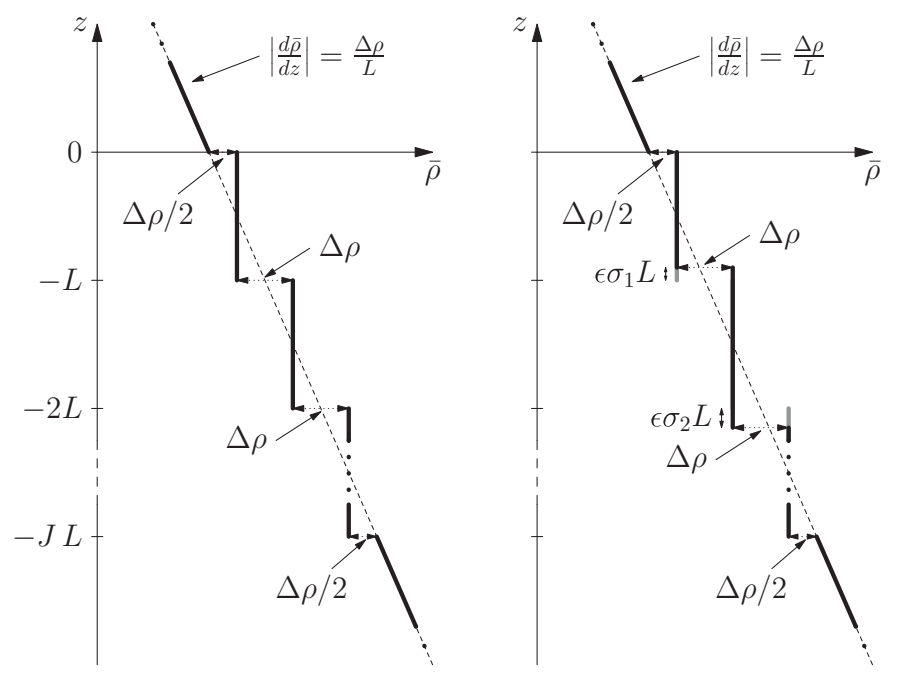

FIG. 1. Schematics showing the density profiles (thick black lines) of a staircase with $J$ equal steps of depth $L$ (left) and a staircase with $J$ irregular steps having mean depth $L$ (right). In both plots the diagonal light dashed line indicates the mean stratification in the absence of a staircase. The extent of the thick gray line segments on the right indicate the change in the depth of the density jump from the case of equal-depth steps.

calculation for several realizations of $\left\{\sigma_{j}\right\}$ provides a means to test the range of extension of the analytic predictions to more realistic oceanographic circumstances.

Generally, the structure of disturbances in nonuniformly stratified fluid is cast in terms of the stream function by $\psi(x, z, t)=\hat{\psi}(z) \exp [\imath(k x-\omega t)]$, in which $k$ and $\omega$ are the horizontal wave number and frequency, respectively, and it is understood that the actual stream function is the real part of the right-hand expression. The (possibly complex) vertical structure function $\hat{\psi}$ satisfies [13]

$$
\frac{d^{2} \hat{\psi}}{d z^{2}}+k^{2}\left(\frac{N^{2}-\omega^{2}}{\omega^{2}-f^{2}}\right) \hat{\psi}=0
$$

in which $f$ is the (constant) Coriolis parameter.

Above and below the staircase (where $N=N_{0}$ ) and within each step (where $N=0$ ), (2) can be solved explicitly. Arbitrarily assuming that internal waves are incident upon the staircase from above, the solution for prescribed $k, \omega$, and incident stream-function amplitude $A_{0}$ is

$$
\hat{\psi}(z)= \begin{cases}A_{0} e^{l m z}+B_{0} e^{-l m z}, & z>0 \\ A_{j} e^{\gamma[z+L(j-1 / 2)]}+B_{j} e^{-\gamma[z+L(j-1 / 2)]}, & -L\left(j+\epsilon \sigma_{j}\right)<z<-L\left(j-1+\epsilon \sigma_{j-1}\right), j=1,2, \ldots, J \\ A_{J+1} e^{l m[z+L J]}, & z<-J L,\end{cases}
$$

in which

$$
m=k \sqrt{\left(N_{0}^{2}-\omega^{2}\right) /\left(\omega^{2}-f^{2}\right)}
$$

is the vertical wave number of the incident wave and

$$
\gamma=k \sqrt{\omega^{2} /\left(\omega^{2}-f^{2}\right)}
$$

is the inverse $e$-folding depth of the evanescent disturbances within the individual uniform-density steps of the staircase. The addition of $L(j-1 / 2)$ in the exponents of (3) is unnecessary because they could be rolled into the $A_{j}$ and $B_{j}$ coefficients. They are included, however, in order to take advantage of symmetry in finding analytic and numerical solutions. 
Provided $f \leqslant \omega \leqslant N_{0}$, the wave is indeed propagating in the uniformly stratified regions surrounding the staircase. The definition of $m$, being positive, is consistent with the incident wave having downward group velocity. The amplitude of the reflected wave is $B_{0}$. By causality, only a downward-propagating wave, with amplitude $A_{J+1}$, can exist in the stratified region below the staircase.

The $2 J+3$ coefficients in Eq. (3) are related through the requirement that the pressure and vertical velocity are continuous. This amounts to the requirement that $\hat{\psi}$ and $d \hat{\psi} / d z-g \frac{\bar{\rho}}{\rho_{00}} \frac{k^{2}}{\omega^{2}} \hat{\psi}$ are continuous [13]. Applied to each of the $J+1$ interfaces, this gives $2 J+2$ equations in $2 J+3$ unknowns. In principle, these can be solved to relate each coefficient to the incident amplitude $A_{0}$. In particular, to find the transmission coefficient it is necessary to find $A_{J+1}$ as a function of $A_{0}$.

The transmission coefficient itself is defined to be the ratio of transmitted to incident energy flux [17]. Because the background is stationary and the stratification is the same above and below the staircase, the transmission is given simply by

$$
T=\left|\frac{A_{J+1}}{A_{0}}\right|^{2} .
$$

The reflection coefficient is given by $R=\left|B_{0} / A_{0}\right|^{2}$. However, in the absence of energy sources such as background shear, it is expected from conservation of energy that $R=1-T$.

\section{ANALYTIC SOLUTION}

\section{A. Finding coefficients}

In the special case of steps having equal depth, it is possible to find explicit analytic solutions by taking advantage of symmetry. After some manipulation the interface conditions give the following sequence of equations:

$$
\begin{aligned}
A_{0} & =\frac{1}{2} \Delta^{1 / 2}\left[1-\frac{l}{M}(1-\Gamma)\right] A_{1}+\frac{1}{2} \Delta^{-1 / 2}\left[1+\frac{l}{M}(1+\Gamma)\right] B_{1}, \\
A_{j} & =\Delta(1-\Gamma) A_{j+1}-\Gamma B_{j+1}, \\
B_{j} & =\Gamma A_{j+1}+\Delta^{-1}(1+\Gamma) B_{j+1}, \\
A_{J} & =\frac{1}{2} \Delta^{1 / 2}[1-\Gamma+\imath M] A_{J+1}, \\
B_{J} & =\frac{1}{2} \Delta^{-1 / 2}[1+\Gamma-\imath M] B_{J+1},
\end{aligned}
$$

in which the middle pair of equations is evaluated for $j=1,2, \ldots, J-1$ and, for convenience, the following nondimensional quantities have been defined:

$$
\begin{aligned}
& \Delta \equiv \exp (\gamma L), \\
& M \equiv m / \gamma=\sqrt{\frac{N_{0}^{2}}{\omega^{2}}-1,} \\
& \Gamma \equiv \frac{g^{\prime} k^{2}}{2 \gamma \omega^{2}}=\frac{1}{2} k L \frac{N_{0}^{2}}{\omega^{2}} \sqrt{1-\frac{f^{2}}{\omega^{2}}} .
\end{aligned}
$$

The results may be combined to get a formula for $A_{0}$ in terms of $A_{J+1}$ alone:

$$
A_{0}=\frac{1}{4 M}\left[V_{L}^{T} \mathcal{C}^{J-1} V_{R}\right] A_{J+1}
$$


in which the superscript $T$ denotes the transpose and the left and right vectors are, respectively,

$$
\begin{aligned}
V_{L} & \equiv\left(\begin{array}{c}
\Delta^{1 / 2}[M-\imath(1-\Gamma)] \\
\Delta^{-1 / 2}[M+\imath(1+\Gamma)]
\end{array}\right), \\
V_{R} & \equiv\left(\begin{array}{c}
\Delta^{1 / 2}[1-\Gamma+\imath M] \\
\Delta^{-1 / 2}[1+\Gamma-\imath M]
\end{array}\right)
\end{aligned}
$$

and the matrix $\mathcal{C}$ is

$$
\mathcal{C}=\left(\begin{array}{cc}
\Delta(1-\Gamma) & -\Gamma \\
\Gamma & \Delta^{-1}(1+\Gamma)
\end{array}\right)
$$

\section{B. Transmission across one step}

In the special case of a single mixed layer $(J=1)$, the expression reduces to

$$
A_{0}=\frac{1}{4 M}\left(2 \Delta M(1-\Gamma)+2 \Delta^{-1} M(1+\Gamma)+\imath\left\{\Delta\left[M^{2}-(1-\Gamma)^{2}\right]-\Delta^{-1}\left[M^{2}-(1+\Gamma)^{2}\right]\right\}\right) A_{2} .
$$

Hence, in the determination of the transmission coefficient, $T$, one finds

$$
T^{-1}=\left|\frac{A_{0}}{A_{2}}\right|=1+\frac{1}{16 M^{2}}\left(\delta_{+}-\delta_{-}\right)^{2},
$$

in which

$$
\begin{aligned}
& \delta_{+} \equiv \Delta\left[(1-\Gamma)^{2}+M^{2}\right], \\
& \delta_{-} \equiv \Delta^{-1}\left[(1+\Gamma)^{2}+M^{2}\right] .
\end{aligned}
$$

Using the expressions in Eq. (8), the transmission coefficient can be written explicitly in terms of the relative horizontal wave number $k L$ and by frequency, which is given implicitly in terms of the quantity

$$
\Theta=\tan ^{-1}(m / k)=\tan ^{-1} \sqrt{\frac{N_{0}^{2}-\omega^{2}}{\omega^{2}-f^{2}}} .
$$

Physically, $\Theta$ represents the angle between the vertical and the constant-phase lines of the incident and transmitted waves. In the circumstance $f=0$, the formula for transmission across a single well-mixed region is recovered [16]:

$$
T=\left\{1+\left[\frac{\sinh (k L)}{\sin 2 \Theta}\left(1+\frac{(k L)^{2}}{\cos ^{2} \Theta}-k L \operatorname{coth} k L\right)\right]^{2}\right\}^{-1} .
$$

This is plotted in Fig. 2(a). As expected, the transmission is close to unity if the horizontal wavelength is much greater than the mixed-layer depth $(k L \ll 1)$. For nonhydrostatic waves, which have frequency close to $N_{0}$, transmission can be large even with $k L$ of order unity. A transmission spike occurs for values $k L$ and $\omega / N_{0}$ where incident vertically propagating internal waves are resonant with interfacial waves on either flank of the mixed region [16]. In particular, this results in perfect transmission for incident waves with $\omega \simeq N_{0}$ and $k L \simeq 2.4$.

In the case with a nonzero Coriolis parameter, the explicit formula for $T$ in terms of $k L$ and $\Theta$ can be found from (12), but the expression is much more complicated. The result in the case $f=0.1 N_{0}$ is plotted in Fig. 2(b). (Although characteristically in the ocean at midlatitudes $f \simeq 0.01 N_{0}$, throughout this paper results are shown with $f=0.1 N$ so that the change in the transmission for $\omega \simeq f$ is clearer.) No transmission occurs if $\omega<f$ because the incident waves would themselves be evanescent in this case. For $\omega>f$, the transmission plot differs from that with $f=0$ only for inertia gravity waves, which have frequency very close to $f$. We find there is negligible effect of $f$ upon the predicted transmission if $\omega>1.5 f$. 

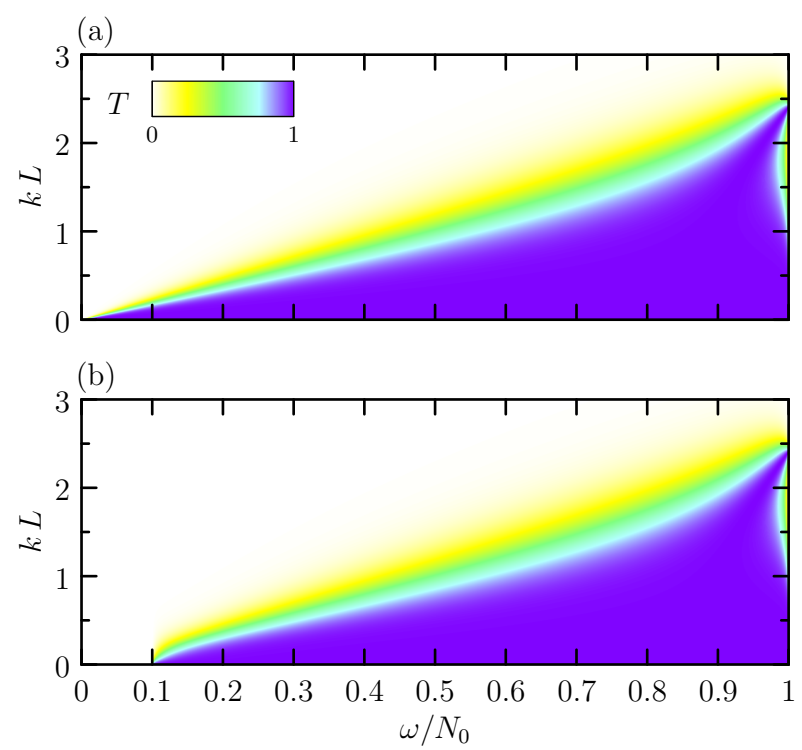

FIG. 2. Transmission through a single mixed region in cases with (a) no background rotation $f=0$ and (b) $f=0.1 N_{0}$. The color scale for both plots is indicated at the top left in (a).

\section{Transmission across $J$ steps}

In the case of a staircase with more than one step $(J>1)$, an explicit formula for the transmission can be found by diagonalizing the matrix $\mathcal{C}$ in Eq. (11). This matrix has determinant equal to one, meaning that the passage of waves through the staircase changes the phase relationship between coefficients $A_{j}$ and $B_{j}$, but does not increase or decrease the magnitude of the disturbances. The (possibly complex) eigenvalues of $\mathcal{C}$ are

$$
\lambda_{ \pm}=b_{+} \pm b_{0}
$$

in which

$$
b_{+} \equiv \frac{1}{2}\left[\Delta(1-\Gamma)+\Delta^{-1}(1+\Gamma)\right]
$$

and

$$
b_{0} \equiv \sqrt{b_{+}^{2}-1} .
$$

Thus we can write $\mathcal{C}=P \Lambda P^{-1}$, in which

$$
\begin{gathered}
\Lambda=\left(\begin{array}{cc}
\lambda_{+} & 0 \\
0 & \lambda_{-}
\end{array}\right), \\
P=\left(\begin{array}{cc}
\frac{1}{\Gamma}\left(b_{-}+b_{0}\right) & \frac{1}{\Gamma}\left(b_{-}-b_{0}\right) \\
1 & 1
\end{array}\right),
\end{gathered}
$$

and

$$
P^{-1}=\frac{\Gamma}{2 b_{0}}\left(\begin{array}{cc}
1 & -\frac{1}{\Gamma}\left(b_{-}-b_{0}\right) \\
-1 & \frac{1}{\Gamma}\left(b_{-}+b_{0}\right)
\end{array}\right),
$$

in which

$$
b_{-} \equiv \frac{1}{2}\left[\Delta(1-\Gamma)-\Delta^{-1}(1+\Gamma)\right] .
$$


Thus an explicit expression can be found for $\mathcal{C}^{J-1}$,

$$
\mathcal{C}^{J-1}=P \Lambda^{J-1} P^{-1}=\frac{1}{b_{0}}\left(\begin{array}{cc}
b_{-} \Lambda_{-}+b_{0} \Lambda_{+} & -\Gamma \Lambda_{-} \\
\Gamma \Lambda_{-} & -b_{-} \Lambda_{-}+b_{0} \Lambda_{+}
\end{array}\right),
$$

in which

$$
\Lambda_{ \pm}=\frac{1}{2}\left[\lambda_{+}^{J-1} \pm \lambda_{-}^{J-1}\right]
$$

This result can be put into (9). Thus, after significant algebra, the transmission coefficient is found to be

$$
T=\left[1+\left(\frac{\delta_{+} \Gamma_{+}+\delta_{-} \Gamma_{-}+2 \delta_{0} \Gamma\left|\Lambda_{-}\right|}{4 M\left|b_{0}\right|}\right)^{2}\right]^{-1}
$$

in which $\delta_{ \pm}$is given by (13), $\delta_{0}=\Gamma^{2}-1+M^{2}$, and $\Gamma_{ \pm}=b_{-}\left|\Lambda_{-}\right| \pm\left|b_{0}\right| \Lambda_{+}$.

Although complicated, the explicit form of $T$ in Eq. (24) makes it amenable to asymptotic analyses. For now, it is noted that the form of $T$ guarantees that the transmission lies between one and zero as the squared expression in Eq. (24) lies between zero and infinity, respectively. Also, it is straightforward to check that if $J=1$ in Eq. (24), the result reduces to the transmission given by (12).

\section{Bounds on transmission}

Figure 3 shows the transmission coefficient for waves passing through two, five, and ten steps. All three plots were constructed by taking $f=0.1 N_{0}$. However, as in Fig. 2, the transmission is found to be approximately the same if $f=0$ except for $\omega \lesssim 1.5 f$, in which case the transmission drops off to zero at the low-frequency cutoff $\omega=f$.

As the number of steps increases, there is an increasing number of transmission peaks apparent particularly for $\omega \lesssim N_{0}$. These peaks propagate back to lower $\omega$, though they are not so apparent for small $k L$ because the transmission remains close to 1 . However, for $\omega \gtrsim f$ the transmission peaks are clearly apparent once more. If there are $J$ steps, the number of transmission peaks, including that at $k L=0$, equals $J$ for $J>3$.

To illustrate this more clearly, Fig. 4 shows values of the transmission coefficient as a function of $k L$ with $\omega / N_{0}$ fixed and computed for $J=5,10$, and 20 steps. Focusing on any one plot, it is obvious that doubling the number of steps doubles the number of transmission spikes. Where the peaks occur for $J=5$ they also occur for $J=10$ and 20. However, the widths of the peaks become narrower. Whatever the value of $J$, the transmission is negligibly small if $k L$ exceeds a cutoff, which is larger as $\omega / f$ increases. Finally, the difference between a transmission peak and an adjacent transmission low is greater if $\omega$ is closer to $f$ or if $k L$ is closer the cutoff relative wave number.

Some of these observations can be quantified by examination of the analytic solution. In particular, the bounds on the region in $k L-\omega / N_{0}$ space for which the transmission spikes occur are given by the condition that the discriminant of the eigenvalues given by (15) is zero: transmission spikes occur where $\lambda_{ \pm}$is complex valued; transmission is negligible if $k L$ is large and $\lambda_{ \pm}$are real valued. The condition $b_{0}=0\left(\left|b_{+}\right|=1\right)$ in Eq. (17) becomes the following when written in terms of the relative horizontal wave number and frequency:

$$
\frac{\omega}{N_{0}}=\frac{k L}{\sqrt{2 \gamma L}} \sqrt{\operatorname{coth}(\gamma L / 2)} \quad \text { or } \quad \frac{\omega}{N_{0}}=\frac{k L}{\sqrt{2 \gamma L}} \sqrt{\tanh (\gamma L / 2)}
$$

in which $\gamma$ is given by (5). The result involving the hyperbolic cotangent is always greater than or equal to one and so can be ignored. If $\omega \gg f$, then $\gamma \simeq k$, in which case the transmission cutoff is 

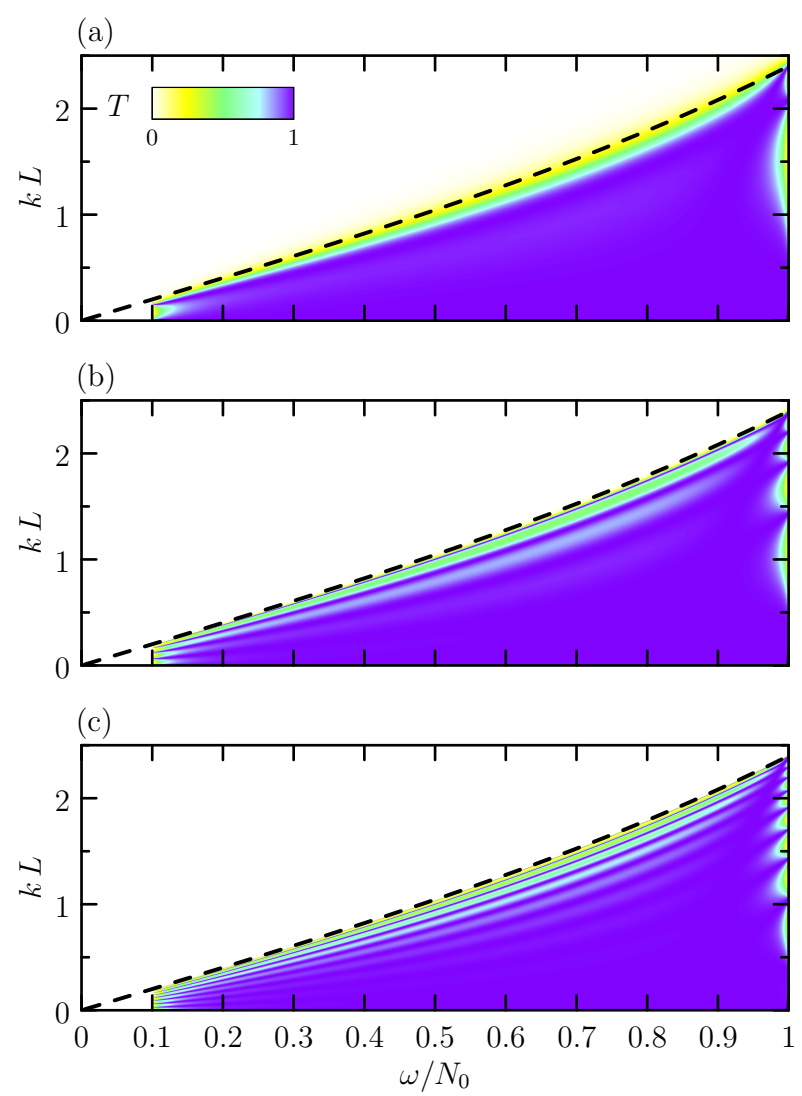

FIG. 3. Same as in Fig. 2 but showing the transmission through a staircase composed of (a) $J=2$, (b) $J=5$, and (c) $J=10$ evenly spaced steps. In all cases $f=0.1 N_{0}$. The dashed lines show the predicted upper bound given by (25) on the region containing the sequence of transmission spikes.

given by

$$
\omega / N_{0}=\sqrt{\frac{k L}{2} \tanh \left(\frac{k L}{2}\right)} .
$$

This curve is drawn in Fig. 3. It is clear that even for $\omega \gtrsim f$, the approximation given by (25) well represents the region between strong and negligible transmission.

The energy-containing internal waves in the ocean are dominantly inertia gravity waves, which have long horizontal scale. Thus we are particularly interested in the transmission occurring in the limit of small $k L$. If $\omega$ is sufficiently large that the effect of $f$ can be neglected, the transmission decreases with increasing $k L \gtrsim 0$ as

$$
T \simeq 1-(k L)^{6}(J-1)^{2} \frac{N_{0}^{4}}{\omega^{4}} \frac{\left(N_{0}^{2} / \omega^{2}-4 / 3\right)^{2}}{64\left[\left(N_{0} / \omega\right)^{2}-1\right]} .
$$

This is accurate to order $k L^{6}$ provided $\omega / N_{0}$ is not close to $(3 / 4)^{1 / 2}$. The sixth power of $k L$ in this expression is an indication that transmission drops off very slowly with increasing, but small, $k L$ if $\omega \gg f$. 

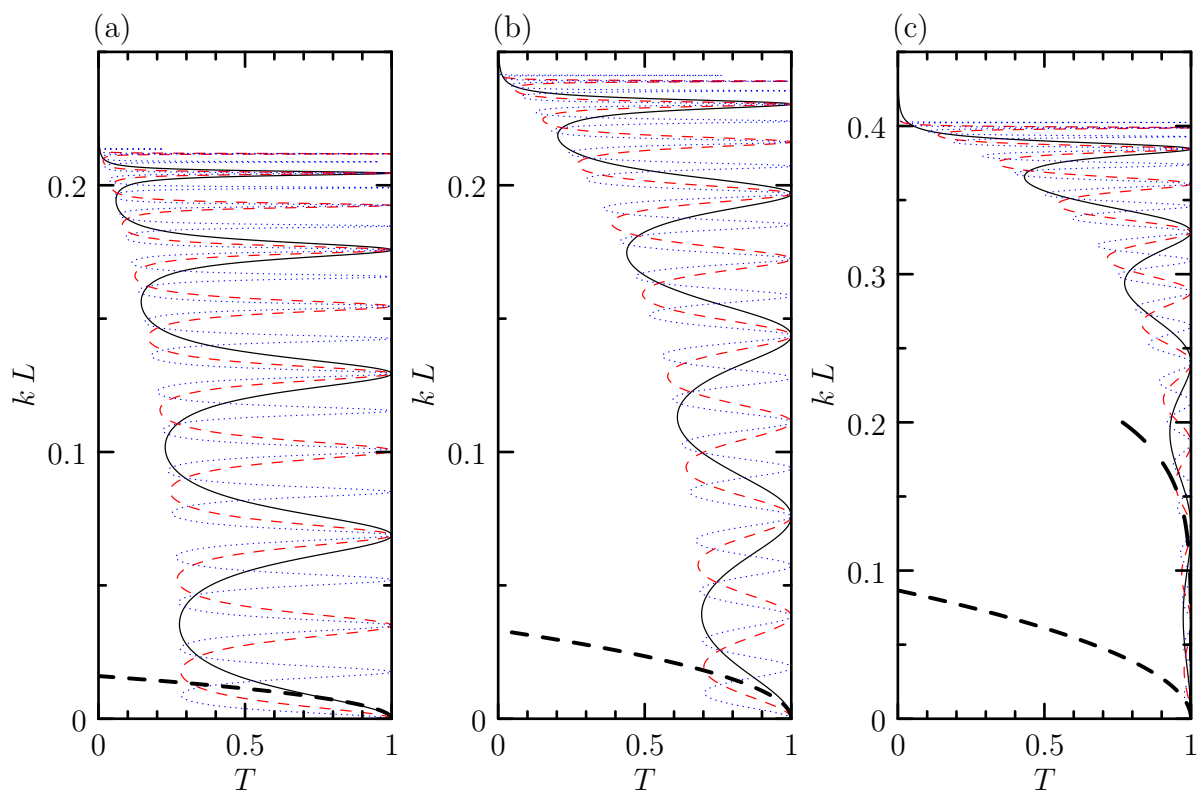

FIG. 4. Transmission as a function of $k L$ for (a) $\omega / N_{0}=0.105(\omega / f=1.05)$, (b) $\omega / N_{0}=0.12(\omega / f=$ $1.2)$, and (c) $\omega / N_{0}=0.2(\omega / f=2.0)$. Plots show transmission through $J=5$ steps (thin solid line), 10 steps (thin red dashed line), and 20 steps (thin blue dotted line). The thick short-dashed lines in each plot gives the approximation (27) to $T$ for $k L \ll 1$ and $\omega \gtrsim f$ for the case $J=5$ and the thick long-dashed line in (c) gives the approximation (26) to $T$ for $k L \ll 1$ and $\omega \gg f$ for the case $J=5$. Note that the vertical scale in (c) is larger than in (a) and (b). In theory all peaks do reach unity, but the finite vertical resolution of the plots $(k L$ is computed in steps of 0.0001) means that the peak at unity is not always captured.

More relevant for inertia gravity waves is the case with $\omega$ close to $f$. Assuming $k D \equiv k L(J-$ $1) \ll 1$ and $k L \ll \sqrt{(\omega / f)^{2}-1}$, the transmission decreases from unity as

$$
T \simeq 1-\frac{1}{4} \frac{N^{2}}{\omega^{2}-f^{2}}(k D)^{2} \simeq 1-\frac{(k D)^{2}}{4 \cos ^{2} \Theta},
$$

in which it has been assumed that $f \ll N_{0}, D=(J-1) L$ is approximately the total depth of the staircase if there are many steps $(J \gg 1)$, and $\Theta$, given by (14), is assumed to be close to $90^{\circ}$ for $\omega \gtrsim f$.

These approximations are superimposed on the plots of $T$ versus $k L$ in Fig. 4 for the case of $J=5$ steps. The approximation given by (27) agrees well with the exact solution in the cases $\omega / f=1.05$ and 1.2 with better agreement to lower values of $T$ if $\omega$ is closer to $f$. In the case $\omega=2 f$, (27) predicts a faster drop-off in $T$ than actually occurs. The exact result is closer to the approximate formula (26), which shows little decrease in transmission for $k L$ up to 0.1 .

These approximate formulas have the potential to make predictions for inertia gravity waves transmitting through density staircases in the ocean. However, before pursuing this, it is necessary to consider how well the analytic results extend to predict transmission through realistic uneven density staircases.

\section{NUMERICAL SOLUTION FOR UNEVEN STEPS}

Although density staircases observed in the ocean typically have a characteristic length scale $L$ determining the depth of each step, there is nonetheless some variation in the extent of each step over the total depth of the staircase. This is represented in the density profile given by (1) in the case 

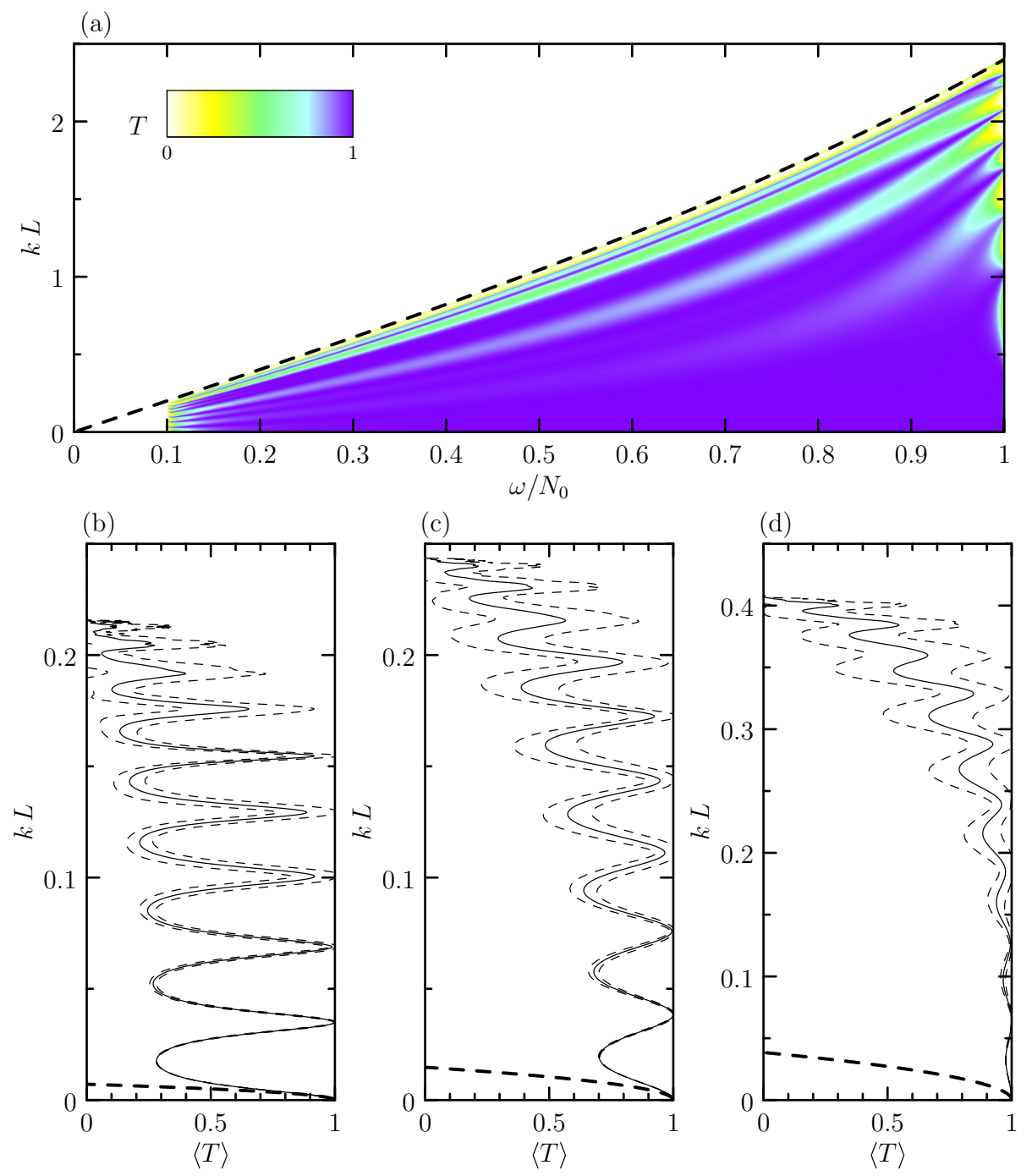

FIG. 5. (a) Same as in Fig. 3(c), but showing transmission through $J=10$ unevenly spaced steps computed from one random realization of $\left\{\sigma_{j}\right\}$ with $\epsilon=0.1$. The average transmission (solid lines) computed in 100 random realizations is plotted as function of $k L$ for $(\mathrm{b}) \omega / N_{0}=0.105(\omega / f=1.05),(\mathrm{c}) \omega / N_{0}=0.12(\omega / f=$ $1.2)$, and (d) $\omega / N_{0}=0.2(\omega / f=2.0)$. The thin-dashed lines in the bottom three plots show the mean plus and minus the standard deviation. The thick-dashed lines show the approximate analytic prediction (27).

$\epsilon>0$. It is not feasible to find analytic solutions for the transmission of internal waves through an uneven staircase. However, it is straightforward to compute the transmission numerically.

The formula relating $A_{0}$ to $A_{J+1}$ is given by (9) but with $\mathcal{C}^{J-1}$ given by the product of the $J-1$ matrices

$$
\Pi_{j=1}^{J-1}\left(\begin{array}{cc}
\Delta(1-\Gamma) & -\Gamma \Delta^{2 \epsilon \sigma_{j}} \\
\Gamma \Delta^{-2 \epsilon \sigma_{j}} & \Delta^{-1}(1+\Gamma)
\end{array}\right) .
$$

Of course this reduces to $\mathcal{C}^{J-1}$ in the case $\epsilon=0$ and, like $\mathcal{C}$, the product of matrices has determinant equal to unity. For a given set of random values $\left\{\sigma_{j} \mid j=1, \ldots, J-1\right\}$ with $\left|\sigma_{j}\right| \leqslant 1$, the product of matrices is computed iteratively to give a $2 \times 2$ matrix with real-valued elements. The result 
is inserted in Eq. (9), the real and imaginary components are separated out, and the transmission coefficient is then computed from the inverse of the sum of the squares of the real and imaginary parts of $A_{0} / A_{J+1}$. As a test of the efficacy of this method, it is confirmed that the procedure reproduces the analytic solution (24) for $\epsilon=0$.

For comparison with Fig. 3(c), Fig. 5(a) plots the transmission coefficient for a staircase with $J=10$ unevenly spaced steps in the case $f=0.1 N_{0}$. Qualitatively, the plots appear similar with transmission spikes occurring below the bound predicted by (25). The obvious discrepancy between this transmission plot and the analytic solution is that the spacing between the peak transmission curves is more irregular. Just for what values of $k L$ the transmission peaks occur more closely together or further apart depends upon the set of random values $\left\{\sigma_{j}\right\}$ that determines the depths of each step relative to the mean depth $L$.

The plots of transmission versus $k L$ for three different frequencies, shown in Figs. 5(b)-5(d), result from computing the transmission for 100 different realizations of the set of random variables $\left\{\sigma_{j}\right\}$ and then averaging the results. The solid line in each plot is the mean transmission and the dashed lines plot the standard deviation above and below the mean.

Although the variation of the transmission becomes larger as $k L$ approaches the bound given by (25), it is obvious that the transmission is insensitive to the variation in step sizes if $k L$ is very small. In particular, the value of the transmission between 0.6 and 1 is well predicted for $f<\omega \lesssim 1.05 f$.

In a separate analysis, the transmission was computed across a staircase in which both the step size and density jump between steps were varied. Imagining that the staircase developed from a linear density profile, the density in each step was taken to be the average of that density profile over the extent of each step. Thus the density in step $j$ was taken to be $\rho_{j}=\rho_{00}+\Delta \rho\left[j-1 / 2+\epsilon\left(\sigma_{j}+\right.\right.$ $\left.\left.\sigma_{j-1}\right)\right]$ and the density jump between step $j+1$ and $j$ was $\Delta \rho_{j}=\Delta \rho\left[1+\epsilon\left(\sigma_{j+1}-\sigma_{j-1}\right) / 2\right]$ for $j=1,2, \ldots, J$. The resulting computation of the transmission coefficient has the form of (9) with $\mathcal{C}^{J-1}$ replaced by (28) and, in this result, having $\Gamma$ replaced by $\Gamma_{0}=\Gamma\left[1+\epsilon \sigma_{1}\right]$ in the $V_{L}$ vector, $\Gamma$ replaced by $\Gamma_{J}=\Gamma\left[1-\epsilon \sigma_{J-1}\right]$ in the $V_{R}$ vector, and $\Gamma$ replaced by $\Gamma_{j}=\Gamma\left[1+\epsilon\left(\sigma_{j+1}-\sigma_{j-1}\right) / 2\right]$ in the $\mathcal{C}$ matrix for $j=1,2, \ldots, J-1$.

The results (not shown) of computing the transmission for several stochastic realizations and averaging are qualitatively similar to those shown in Fig. 5. Importantly, the transmission remains insensitive to the variation of both step sizes and density jumps if $k L$ is very small.

\section{DISCUSSION AND CONCLUSION}

An analytic prediction was made for the transmission of internal waves through a staircase density profile with an arbitrary number of evenly spaced steps. Compared with the numerical solution computed for waves passing through an uneven staircase, the analytic predictions for the wave-number cutoff bounding the region between high and low transmission were found to remain accurate. Crucially, the transmission as it falls from unity for $k L$ increasing from zero was found to be well predicted even for waves passing through an uneven staircase. In particular, for inertia gravity waves having $\omega-f \ll f, k L \ll \sqrt{(\omega / f)^{2}-1}$, and $k D \ll 1$ (with $L$ and $D$, respectively, the depths of each step and the whole staircase), the transmission is given approximately by (27).

In practice, (27) is found to well predict the transmission as it falls to 0.6 if $f<\omega \lesssim 1.05 f$. Thus we can invert this approximate equation to answer the following question: What is the lower bound on the horizontal wavelength of incident inertia gravity waves such that their transmission lies between 1 and $1-R$, with $R$ (the reflection coefficient) small? Denoting the lower bound on the wavelength of transmitting waves by $\lambda_{T l}$, we have

$$
\lambda>\lambda_{T l} \simeq \frac{\pi D}{2}\left[\frac{\omega^{2}-f^{2}}{N_{0}^{2}} R\right]^{-1 / 2} .
$$


In particular, taking $f=0.01 N_{0}, \omega=1.05 f_{0}$, and $R=0.1$, the lower bound is $\lambda_{T l} \simeq 1.6 \times 10^{3} \mathrm{D}$. So for inertia gravity waves incident upon a staircase of total depth $30 \mathrm{~m}$ [22], the waves transmit with little reflection if their horizontal wavelength is larger than $\simeq 50 \mathrm{~km}$. This is on the horizontal scale of internal waves generated by storms. Thus, consistent with the assertion of Ghaemsaidi et al. [22], this work predicts that density staircases may act as an effective filter allowing waves with longer horizontal wavelength and larger frequencies to transmit through to the abyss. Shorter waves may reflect or transmit substantially. According to (25), strong reflection of inertia gravity waves is certain if $k L>2 f / N_{0}$. Taking $f \simeq 0.01 N_{0}$, the upper bound on the horizontal wavelength of waves that must reflect from a staircase with 1-m-deep steps is $\lambda_{R u} \lesssim \pi L N_{0} / f \simeq 300 \mathrm{~m}$.

\section{ACKNOWLEDGMENT}

This work was supported by funding through the Discovery Grant program of the Natural Sciences and Engineering Research Council of Canada.

[1] R. W. Schmitt, Double diffusion in oceanography, Annu. Rev. Fluid Mech. 26, 255 (1994).

[2] O. M. Johannessen and O. S. Lee, Thermohaline staircase structure in the Tyrrhenian sea, Deep-Sea Res. 21, 629 (1974).

[3] R. Molcard and A. J. Williams, Deep-stepped structure in the Tyrrhenian sea, Mem. Soc. R. Sci. Liege, 6th Ser. 8, 191 (1975).

[4] P. A. Mazeika, Subsurface mixed layers in the northwest tropical Atlantic, J. Phys. Oceanogr. 4, 446 (1974).

[5] R. B. Lambert and W. Sturges, A thermohaline staircase and vertical mixing in the thermocline, Deep-Sea Res. 24, 211 (1977).

[6] R. W. Schmitt, H. Perkins, J. D. Boyd, and M. C. Stalcup, C-SALT: An investigation of the thermohaline staircase in the western tropical North Atlantic, Deep-Sea Res. 34, 1655 (1987).

[7] R. A. Woodgate, K. Aagaard, J. H. Swift, W. M Smethie, Jr., and K. K. Falkner, Atlantic water circulation over the Mendeleev Ridge and Chukchi Borderland from thermohaline intrusions and water mass properties, J. Geophys. Res. 112, C02005 (2007).

[8] L. Rainville and P. Winsor, Mixing across the Arctic Ocean: Microstructure observations during the Beringia 2005 Expedition, Geophys. Res. Lett. 35, L08606 (2008).

[9] M.-L. Timmermans, J. Toole, R. Krishfield, and P. Winsor, Ice-Tethered Profiler observations of the double-diffusive staircase in the Canada Basin thermocline, J. Geophys. Res. 113, C00A02 (2008).

[10] M.-L. Timmermans, S. Cole, and J. Toole, Horizontal density structure and restratification of the Arctic Ocean surface layer, J. Phys. Oceanogr. 42, 659 (2012).

[11] L. Rainville and R. A. Woodgate, Observations of internal wave generation in the seasonally ice-free Arctic, Geophys. Res. Lett. 36, L23604 (2009).

[12] L. Rainville, C. M. Lee, and R. A. Woodgate, Impact of wind-driven mixing in the Arctic Ocean, Oceanography 24, 136 (2011).

[13] B. R. Sutherland, Internal Gravity Waves (Cambridge University Press, Cambridge, 2010), p. 378.

[14] E. Salusti, Internal waves on a deep-stepped marine structure, Deep-Sea Res. 25, 947 (1978).

[15] C. Eckart, Internal waves in the ocean, Phys. Fluids 4, 791 (1961).

[16] B. R. Sutherland and K. Yewchuk, Internal wave tunnelling, J. Fluid Mech. 511, 125 (2004).

[17] G. L. Brown and B. R. Sutherland, Internal wave tunneling through non-uniformly stratified shear flow, Atmos. Ocean 45, 47 (2007).

[18] J. T. Nault and B. R. Sutherland, Beyond ray tracing for internal waves. Part I: Small-amplitude anelastic waves, Phys. Fluids 20, 106601 (2008). 


\section{INTERNAL WAVE TRANSMISSION THROUGH A ...}

[19] M. Mathur and T. Peacock, Internal wave beam propagation in non-uniform stratifications, J. Fluid Mech. 639, 133 (2009).

[20] M. Mathur and T. Peacock, Internal Wave Interferometry, Phys. Rev. Lett. 104, 118501 (2010).

[21] K. Gregory and B. R. Sutherland, Transmission and reflection of internal wave beams, Phys. Fluids 22, 106601 (2010).

[22] S. Ghaemsaidi, H. V. Dosser, L. Rainville, and T. Peacock, The impact of multiple layering on internal wave transmission, J. Fluid Mech. 789, 589 (2016).

[23] G. L. Brown, A. B. G. Bush, and B. R. Sutherland, Beyond ray tracing for internal waves. Part II: Finite-amplitude effects, Phys. Fluids 20, 106602 (2008). 